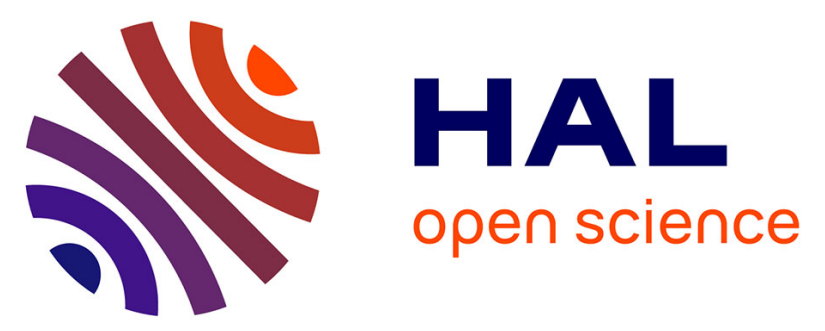

\title{
A multi-time-scale strategy for multiphysics problems: Application to poroelasticity
}

\author{
David Dureisseix, Pierre Ladevèze, David Néron, Bernard A. Schrefler
}

\section{To cite this version:}

David Dureisseix, Pierre Ladevèze, David Néron, Bernard A. Schrefler. A multi-time-scale strategy for multiphysics problems: Application to poroelasticity. International Journal for Multiscale Computational Engineering, 2003, 1 (4), pp.387-400. 10.1615/IntJMultCompEng.v1.i4.50 . hal-00321779

\section{HAL Id: hal-00321779 \\ https://hal.science/hal-00321779}

Submitted on 13 Jan 2020

HAL is a multi-disciplinary open access archive for the deposit and dissemination of scientific research documents, whether they are published or not. The documents may come from teaching and research institutions in France or abroad, or from public or private research centers.
L'archive ouverte pluridisciplinaire HAL, est destinée au dépôt et à la diffusion de documents scientifiques de niveau recherche, publiés ou non, émanant des établissements d'enseignement et de recherche français ou étrangers, des laboratoires publics ou privés.

\section{다)(1) $(5$}

Distributed under a Creative Commons Attribution - NonCommercial| 4.0 International 


\title{
A multi-time-scale strategy for multiphysics problems: application to poroelasticity
}

\author{
David Dureisseix* ${ }^{*}$ Pierre Ladevèze ${ }^{\dagger}$, David Néron ${ }^{\dagger}$ and Bernhard A. Schrefler ${ }^{\ddagger}$
}

\author{
* LMGC (University Montpellier 2 / CNRS) \\ CC 048, place Eugène Bataillon, F-34095 Montpellier CEDEX 5, France \\ ${ }^{\dagger}$ LMT-Cachan (ENS Cachan / CNRS / University Paris 6) \\ 61, avenue du Président Wilson, F-94235 Cachan CEDEX, France \\ ${ }^{\ddagger}$ Department of Structural and Transportation Engineering (University of Padova) \\ Via Marzolo 9, I-35131 Padova, Italy
}

\begin{abstract}
Usually, multiphysics phenomena and coupled-field problems lead to computationally intensive structural analysis. Strategies to keep these problems computationally affordable are of special interest. For coupled fluidstructure problems, for instance, partitioned procedures and staggered algorithms are often preferred to direct analysis.

In a previous paper, a new strategy derived from the LArge Time INcrement (LATIN) method was described. This strategy was applied to the consolidation of saturated porous soils, which is a highly coupled fluid-solid problem. The feasibility of the method and the comparison of its performance with that of a standard partitioning scheme (the so-called ISPP method) was presented.

Here, we go one step further and use the LATIN method to take into account the different time scales which usually arise from the different physics. We propose a multi-time-scale strategy which improves the existing method.

This is a post-print of an article published in its original version as: David Dureisseix, Pierre Ladevèze, David Néron, Bernhard A. Schrefler, A multi-time-scale strategy for multiphysics problems: application to poroelasticity, International Journal for Multiscale Computational Engineering 1(4):387-400, Begell House, 2004. DOI: 10.1615/IntJMultCompEng.v1.i4.50 ISSN: 1543-1649
\end{abstract}

Keywords: multiphysics, multi-time-scale, coupled fields, LATIN, porous media, consolidation

\section{INTRODUCTION}

For coupled multiphysics problems such as fluid-structure interaction, from the point of view of computational efficiency, partitioned procedures and staggered algorithms [FP80, FG88, LSS91, OM95, PFL95, SL98, FL00] are often preferred to direct analysis (also called the monolithic approach). Moreover, partitioning strategies enable one to use different analyzers for different subsystems and help keep the software manageable.

Recently, a mixed domain decomposition strategy based on the Large Time INcrement method (LATIN), see [Lad99], for parallel computing environments has been developed [DL98]. The use of a multilevel approach with a built-in homogenization procedure makes this strategy suitable for highly heterogeneous problems [LD00]. An approach suitable for multiphysics problems has been designed on the same premises and applied to the consolidation of saturated porous soils, which is a typical example of a highly coupled fluid-structure interaction problem.

Most of the partitioning strategies for coupled problems rely on splitting the unknowns into groups related to each physics (for instance, the solid part and the fluid part of the problem). Thus, a different solver can be used for each physics. The resolution of the direct coupled problem (the so-called monolithic problem) is replaced by iterations between resolutions of the uncoupled problems and exchanges of information between solvers to recover the coupling parameters.

In this paper, we compare the LATIN approach to the Iterated Standard Staggered Procedure (ISPP [MSV96]), which is one of these standard partitioning schemes. This comparison is made on 1D and 2D problems and is an extension of the first comparison published earlier along with the principles of the proposed multiphysics strategy in [DLS03].

An interesting new aspect concerns the behavior of the algorithm when the complexity of the loading vs. time increases. Indeed, in many cases, the cost of achieving convergence with the method is related to this complexity. 
Also, using different solvers for different physics leads to the question of the independent modeling and discretization of each physical phenomenon. Usually, in multiphysics phenomena, different time scales must be dealt with simultaneously. It is advantageous to assign its own time discretization to each solver. Therefore, in this paper, we propose an improved procedure which consists of using the LATIN method as a multi-time-scale strategy to take into account these different time scales.

Finally, in [DLS03], an ad hoc radial loading approximation of the kinematic unknowns was proposed. Here, we present a more complete description of both kinematic and static quantities.

The numerical tests deal with the consolidation of saturated porous soils; the reference problem is reviewed in Section 2 In Section 3 for the sake of completeness, we present a brief summary of the principles of the LATIN method for multiphysics problems and the $2 \mathrm{D}$ numerical test used to evaluate the method against the classical ISPP approach and demonstrate its features with respect to the time complexity of the loading. Section 4 deals with the multi-time-scale approach and its numerical performance on a $1 \mathrm{D}$ consolidation problem. Section 5 presents a strategy of representation of both kinematic and static quantities. Finally, Section 7 presents our conclusions and outlines future studies.

\section{THE REFERENCE PROBLEM}

Let us consider a structure $\Omega$ made of a saturated porous material undergoing small perturbations and isothermal evolution over the time interval $[0, T]$ being studied.

The loading consists of: a prescribed displacement $\underline{U}_{d}$ on part of the boundary $\partial_{1} \Omega$, a traction force $\underline{F}_{d}$ on the complementary part $\partial_{2} \Omega$ of $\partial_{1} \Omega$, a fluid flux $w_{d}$ on another part $\partial_{3} \Omega$ of the boundary and, finally, a prescribed pore pressure $p_{d}$ on the complementary part $\partial_{4} \Omega$ of $\partial_{3} \Omega$ (see Figure 1 ). For the sake of simplicity, we assume that there are no body forces.

For solid quantities, strain is designated by $\varepsilon$ and stress by $\sigma$. For fluid quantities, the pore pressure gradient is designated by $\underline{Z}$ and the opposite of Darcy's velocity by $\underline{W}$; finally, $q$ designates the rate of fluid mass accumulation in each representative elementary volume.

The state of the structure is given by the set of the fields $\mathbf{s}=(\varepsilon, p, \underline{Z}, \boldsymbol{\sigma}, q, \underline{W})$ defined on the whole structure $\Omega$ and on the time interval $[0, T]$ being considered. The problem consists of finding $\mathbf{s}$ in the corresponding space $\mathbf{S}^{[0, T]}$ which verifies, at each time step, the following equations:

- in the solid, compatibility of the strains $\varepsilon$ and equilibrium of the stresses $\sigma$ :

$$
\begin{aligned}
& \underline{U} \in \mathcal{U}^{[0, T]} \quad \text { and } \quad \varepsilon=\varepsilon(\underline{U}) \quad \text { on } \Omega \\
& \underline{\operatorname{div} \sigma}=\underline{0} \quad \text { on } \Omega \quad \text { and } \quad \boldsymbol{\sigma} \underline{n}=\underline{F}_{d} \quad \text { on } \partial_{2} \Omega
\end{aligned}
$$

$\mathcal{U}^{[0, T]}$ being the set of the finite-energy displacement fields on $\Omega \times[0, T]$ equal to $\underline{U}_{d}$ on $\partial_{1} \Omega$;

- in the fluid, flow conservation for Darcy's velocity $-\underline{W}$ :

$$
\begin{array}{r}
p \in \mathcal{P}^{[0, T]} \text { and } \underline{Z}=\underline{\operatorname{grad} p} \quad \text { on } \Omega \\
q=\operatorname{div} \underline{W} \quad \text { on } \Omega \quad \text { and } \underline{W} \cdot \underline{n}=w_{d} \quad \text { on } \partial_{4} \Omega
\end{array}
$$

$\mathcal{P}^{[0, T]}$ being the set of the finite-energy pressure fields on $\Omega \times[0, T]$ equal to $p_{d}$ on $\partial_{3} \Omega$;

- the constitutive relations:

- Hooke's law, which relates the macroscopic stress $\sigma$ with the strain $\varepsilon$ and the pore pressure $p$ such that:

$$
\boldsymbol{\sigma}=\mathbf{D} \boldsymbol{\varepsilon}-b p \mathbf{1}
$$

- Darcy's law, which relates Darcy's velocity to the pore pressure gradient:

$$
\underline{W}=\frac{K}{\mu_{w}} \underline{Z}
$$

- compressibility, which relates the fluid accumulation rate to the pressure rate and couples it with the rate of volume modification:

$$
q=\frac{1}{Q} \dot{p}+b \operatorname{Tr} \dot{\varepsilon}
$$

D is Hooke's tensor of the drained skeleton, $b$ is Biot's coefficient, $K$ is the intrinsic macroscopic permeability and $\mu_{w}$ is the dynamic viscosity of the saturation fluid. Throughout the following sections, the operator $\frac{K}{\mu_{w}} \mathbf{1}$ will be designated by $\mathbf{H}$. Finally, $Q$ is Biot's modulus. 


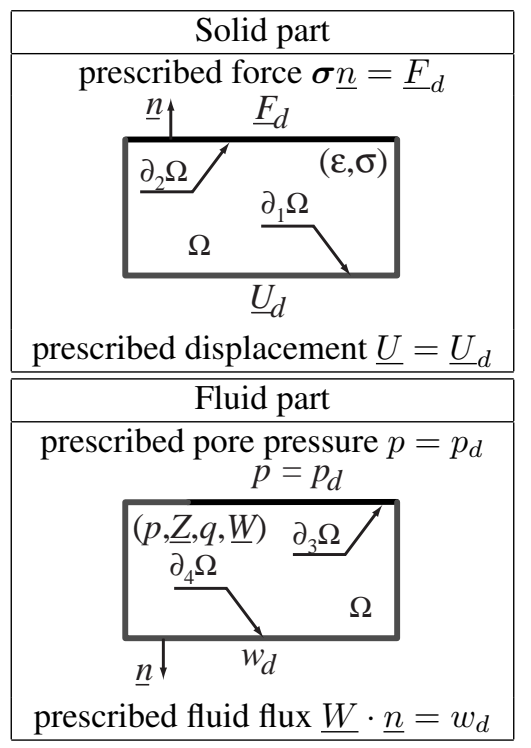

Figure 1: The reference problem

\section{LATIN COMPUTATIONAL STRATEGY}

\subsection{Principles of the method}

The LATIN method is a nonincremental iterative approach originally designed for nonlinear time-dependent problems [Lad99]. However, its principles have also been successfully applied to dynamic problems, postbuckling and domain decomposition, see [BLL00, LBL02, LLD01, LN02, LNL02].

For coupled multiphysics problems, its development was described and examples of its feasibility given in [DLS03]. Only the main principles of the approach will be recalled here. The reader may refer to [DLS03] for further details.

At each iteration, the LATIN method produces an approximation of the solution over the whole domain and over the entire time interval being studied. It is based on three principles:

- The first principle consists of separating the difficulties. For coupled-field problems, a first set of equations, $\mathbf{A}_{\mathbf{d}}$, containing the so-called admissibility conditions $[1 / 2)$ is defined. To avoid having to treat both a global and a coupled problem simultaneously, the remaining equations are grouped into a second set of equations, $\boldsymbol{\Gamma}$; these equations, which are local in the space variables, are the constitutive relations 3445 . The solution, i.e. the set of fields belonging to both $\mathbf{A}_{\mathbf{d}}$ and $\boldsymbol{\Gamma}$, is found using an iterative procedure.

- The second principle of the method consists of using search directions to build approximate solutions of $\mathbf{A}_{\mathbf{d}}$ and $\boldsymbol{\Gamma}$ alternatively until a sufficient level of convergence has been reached. Each iteration consists of 2 stages:

Once an element $\mathbf{s}_{n} \in \mathbf{A}_{\mathbf{d}}$ is known, the local stage at iteration $n+1$ uses an initial search direction to provide an element $\hat{\mathbf{s}}_{n+1 / 2} \in \mathbf{\Gamma}$ :

$$
\begin{aligned}
\left(\hat{\boldsymbol{\sigma}}_{n+1 / 2}-\boldsymbol{\sigma}_{n}\right)+\mathbf{L}\left(\hat{\dot{\boldsymbol{\varepsilon}}}_{n+1 / 2}-\dot{\boldsymbol{\varepsilon}}_{n}\right) & =0 \\
\left(\underline{W}_{n+1 / 2}-\underline{W}_{n}\right)+\mathbf{H}\left(\underline{\hat{Z}}_{n+1 / 2}-\underline{Z}_{n}\right) & =0 \\
\left(\hat{q}_{n+1 / 2}-q_{n}\right)+r\left(\hat{p}_{n+1 / 2}-p_{n}\right) & =0
\end{aligned}
$$

$\mathbf{L}$ and $r$ are two parameters of the method; they do not influence the solution once convergence has been reached. However, their values modify the convergence rate of the algorithm. In dimensional analysis, they can be chosen of the form $\mathbf{L}=t_{m} \mathbf{D}$ and $r=\frac{1}{Q t_{h}}$ where $t_{m}$ and $t_{h}$ are two arbitrary characteristic times.

At each integration point, with the constitutive relations $3 \sqrt[345]{5}$, the local stage leads to the resolution of a small system of ordinary differential equations in the local space variables:

$$
\begin{array}{r}
\mathbf{L} \hat{\dot{\varepsilon}}+\mathbf{D} \hat{\varepsilon}-b \hat{p} \mathbf{1}=\mathbf{A}_{n}(t) \\
\frac{1}{Q} \hat{\dot{p}}+r \hat{p}+b \operatorname{Tr} \hat{\dot{\varepsilon}}=\alpha_{n}(t)
\end{array}
$$

where $\mathbf{A}_{n}=\boldsymbol{\sigma}_{n}+\mathbf{L} \dot{\boldsymbol{\varepsilon}}_{n}$ and $\alpha_{n}=q_{n}+r p_{n}$ are known quantities at the local stage $n+1$ and with the initial conditions on the pressure and strain fields. 
Once an element $\hat{\mathbf{s}}_{n+1 / 2} \in \boldsymbol{\Gamma}$ is known, the linear stage provides an element $\mathbf{s}_{n+1} \in \mathbf{A}_{\mathbf{d}}$. $\mathbf{s}_{n+1}$, which must satisfy the admissibility relations, is sought along a search direction conjugate of the previous one so that the mechanical and hydraulic problems remain uncoupled:

$$
\begin{array}{r}
\left(\boldsymbol{\sigma}_{n+1}-\hat{\boldsymbol{\sigma}}_{n+1 / 2}\right)-\mathbf{L}\left(\dot{\boldsymbol{\varepsilon}}_{n+1}-\hat{\dot{\boldsymbol{\varepsilon}}}_{n+1 / 2}\right)=0 \\
\left(\underline{W}_{n+1}-\underline{\hat{W}}_{n+1 / 2}\right)-\mathbf{H}\left(\underline{Z}_{n+1}-\underline{\hat{Z}}_{n+1 / 2}\right)=0 \\
\left(q_{n+1}-\hat{q}_{n+1 / 2}\right)-r\left(p_{n+1}-\hat{p}_{n+1 / 2}\right)=0
\end{array}
$$

In order to use a finite element approach, the admissibility of $\mathbf{s}_{n+1}$ is expressed using variational formulations. On the one hand, it consists of $\underline{U} \in \mathcal{U}^{[0, T]}$ and $\sigma \in \mathcal{S}^{[0, T]}$ such that:

$$
\forall t \in[0, T], \quad \forall \underline{U}^{\star} \in \mathcal{U}_{0},
$$

$$
\int_{\Omega} \operatorname{Tr}\left[\sigma \varepsilon\left(\underline{U}^{\star}\right)\right] d \Omega=\int_{\partial_{2} \Omega} \underline{F}_{d} \cdot \underline{U}^{\star} d S
$$

where $\mathcal{U}_{0}$ is the set of the finite-energy displacement fields on $\Omega$ which vanish on $\partial_{1} \Omega$. On the other hand, it also consists of $p \in \mathcal{P}^{[0, T]}, q \in \mathcal{Q}^{[0, T]}$ and $\underline{W} \in \mathcal{W}^{[0, T]}$ such that:

$$
\forall t \in[0, T], \quad \forall p^{\star} \in \mathcal{P}_{0}
$$

$$
\int_{\Omega}\left(q p^{\star}+\underline{W} \cdot \underline{\operatorname{grad}} p^{\star}\right) d \Omega=\int_{\partial_{4} \Omega} w_{d} p^{\star} d S
$$

where $\mathcal{P}_{0}$ is the set of the finite-energy pressure fields on $\Omega$ which vanish on $\partial_{3} \Omega$.

The equations 9,10 , define two uncoupled global problems parameterized with time $t$.

The convergence of this approach is guaranteed provided that $\mathbf{L}$ and $r$ are positive definite operators, see [Lad99].

- The third principle uses the fact that the successive approximations are defined over both the entire domain and the entire time interval to represent the solution on a radial loading basis. This last point was detailed in [Lad99] and will be developed in our particular case in a subsequent section. Briefly, this approach enables one to reduce the number of space fields generated and, therefore, the number of global systems to be solved.

In the following sections, the LATIN method without this third principle will be referred to as "without P3"; with an approximation of only the kinematic fields, it will be referred to as "1/2 P3" [DLS03] and with an approximation of both the kinematic and the static fields, it will be referred to as "P3".

\subsection{Test case}

The proposed test case concerns the consolidation of a Berea sandstone soil. The material characteristics in Table 1 were identified in [GRE90].

\begin{aligned} & \hline Porosity $n=0.19 \\ &$ Young's modulus $E=14.4 \mathrm{GPa} \\ &$ Poisson's ratio $\nu=0.2 \\ &$ Biot's modulus $Q=13.5 \mathrm{GPa} \\ &$ Biot's coefficient $b=0.78 \\ &$ Permeability $\frac{K}{\mu_{w}}=210^{-10} \mathrm{~m}^{3} . \mathrm{s} . \mathrm{kg}^{-1} \\ &$\hline\end{aligned}

Table 1: Poro-elastic material characteristics of a water-saturated Berea sandstone

Two different $2 \mathrm{D}$ tests were used to illustrate the behavior of the method. The first test is the same as in [DLS03] and, therefore, we will mention only the conclusions. The second test is dedicated more specifically to the evaluation of the influence of the time complexity of the loading in the different approaches.

In each case, the space discretization was performed using P2 elements (6-node triangles) for the displacement and P1 linear interpolation (also continuous throughout the elements) for the pore pressure, see [ZQTN86, BF91]. The $\theta$-method with linear evolution of the variables in time was used for the time integration. [HKS96 VA81] propose the accuracy condition $\frac{\Delta t}{\Delta \ell^{2}} \geqslant \frac{1}{6 \theta c}$, where $\Delta t$ is the length of a time step, $\Delta \ell$ the size of a spatial element and $c=E \frac{K}{\mu_{w}} \frac{3-2 \nu}{3(1+\nu)(1-2 \nu)}$. Here, since $\theta=1$ throughout, this condition leads to $\frac{\Delta t}{\Delta \ell^{2}} \geqslant 0.048 \mathrm{sm}^{-2}$.

In order to compare the results with the ISPP strategy, let us introduce some notations: $n_{T}$ is the number of time steps in the interval being studied; $n_{S}$ and $n_{F}$ are the numbers of global uncoupled resolutions, i.e. the costly parts of the algorithms, for the solid and for the fluid respectively. 
Convergence is reached when an error $\eta$ has become sufficiently small (less than $1 \%$ ). In order to compare algorithms, this error was computed with respect to a reference solution $\mathbf{s}_{e x}$ obtained with the direct monolithic approach, see [DLS03]. This reference solution was calculated with the same space and time discretizations as for the LATIN and the ISPP methods: thus, both algorithms converge toward this solution and the error $\eta$ tends to zero as the number of iterations increases.

$$
\eta=\sqrt{\eta_{S}^{2}+\eta_{F}^{2}}
$$

with

and

$$
\eta_{S}^{2}=\frac{e_{S}^{2}\left(\mathbf{s}-\mathbf{s}_{e x}\right)}{e_{S}^{2}\left(\mathbf{s}_{e x}\right)+e_{F}^{2}\left(\mathbf{s}_{e x}\right)}
$$

and, with the energy norms:

$$
\eta_{F}^{2}=\frac{e_{F}^{2}\left(\mathbf{s}-\mathbf{s}_{e x}\right)}{e_{S}^{2}\left(\mathbf{s}_{e x}\right)+e_{F}^{2}\left(\mathbf{s}_{e x}\right)}
$$

and

$$
e_{S}^{2}(\mathbf{s})=\frac{1}{2} \int_{0}^{T} \int_{\Omega} \operatorname{Tr}[\varepsilon \mathbf{D} \varepsilon] d \Omega d t
$$

$$
e_{F}^{2}(\mathbf{s})=\frac{1}{2} \int_{0}^{T} \int_{\Omega} p Q^{-1} p d \Omega d t
$$

Note that $\eta_{S}$ and $\eta_{F}$ are respectively the solid and the fluid contributions to the total error $\eta$.

Contrary to the case of weak fluid-structure coupling [FP80], classical staggered schemes, when applied to highly coupled problems such as fluid transfer in porous media, lack consistency [SL98, TS93] TS94]. The ISPP approach regains consistency thanks to sub-cycling between the solid and the fluid solvers at each time step. Then, the number of global resolutions for each solver is $n_{\text {sub }} \times n_{T}$.

In the LATIN method without P3, 2 uncoupled problems are solved at each iteration and at each time step. If $n_{\text {it }}$ is the number of iterations required to reach convergence, the number of global resolutions is $n_{\text {it }} \times n_{T}$ for each solver. In [DLS03], a representation of the unknowns was added to improve performance. This affects only the linear stage, where only one space field for the fluid and one for the solid are combined to build a basis for the solution. Moreover, these fields are generated only when they are required to improve the solution in an automatically adaptive fashion. In this first paper, only the displacement field for the solid and the pore pressure field for the fluid are approximated with a radial loading basis (the approach referred to as 1/2P3).

The results of the first test case (the force-driven problem of Figure 2 were reported in [DLS03]. The time interval was $T=36 \mathrm{~s}$, with $t_{1}=T / 10$ and the pressures were $p_{1}=1.54 \mathrm{GPa}$ and $p_{0}=380 \mathrm{MPa}$; the initial condition was $p(t=0)=p_{0}$.

Using a representation of the unknowns, the LATIN method was found to be computationally competitive and relatively insensitive to the coupling Biot's coefficient $b$. Table 2 shows a summary of the results that were obtained.
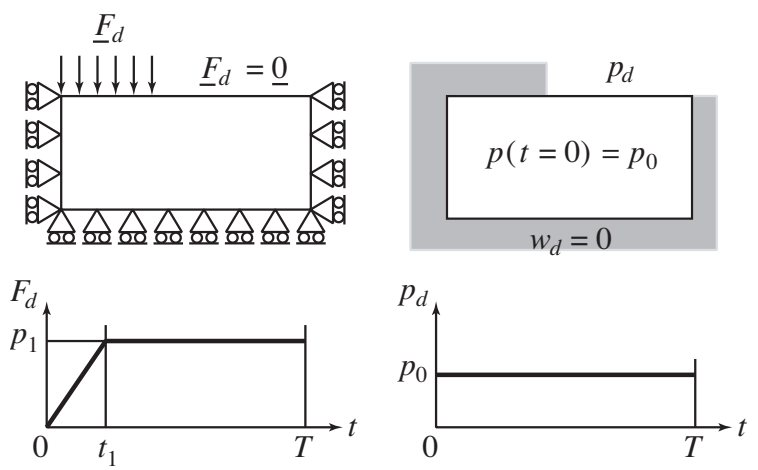

Figure 2: The force-driven test problem (left: prescribed solid quantities, middle: prescribed fluid quantities, right: time evolution of the loading)

The mesh used was the one shown Figure 3 . The same mesh was also used for the second test we propose here. The accuracy criterion is $\left(\frac{\Delta t}{\Delta \ell^{2}}\right)_{\min }=0.075 \mathrm{sm}^{-2}>0.048 \mathrm{sm}^{-2}$, which verifies the previous condition.

In this second test, the calculation of the fluid-flux-driven problem of Figure 4 was performed with three different loading cases. The results are presented in Table 3 The search direction parameters were optimized as in [DLS03] and set to $t_{m}=0.04 t_{c}$ and $t_{h}=0.03 t_{c}$, where $t_{c}=\mu_{w} L^{2} /(Q K)=148 \mathrm{~s}$ is a characteristic time of the problem. 


\begin{tabular}{ccc}
\hline \multirow{2}{*}{ ISPP } & $n_{S}$ & 1,080 \\
& $n_{F}$ & 1,080 \\
& $n_{\text {sub }}$ & 9 \\
\hline \multirow{3}{*}{ LATIN without P3 } & $n_{S}$ & 2,160 \\
& $n_{F}$ & 2,160 \\
& $n_{\text {it }}$ & 18 \\
\hline \multirow{3}{*}{ LATIN with $1 / 2 \mathrm{P} 3$} & $n_{S}$ & 8 \\
& $n_{F}$ & 16 \\
& $n_{\text {it }}$ & 27 \\
\hline
\end{tabular}

Table 2: Number of global resolutions for the force-driven problem with $n_{T}=120$

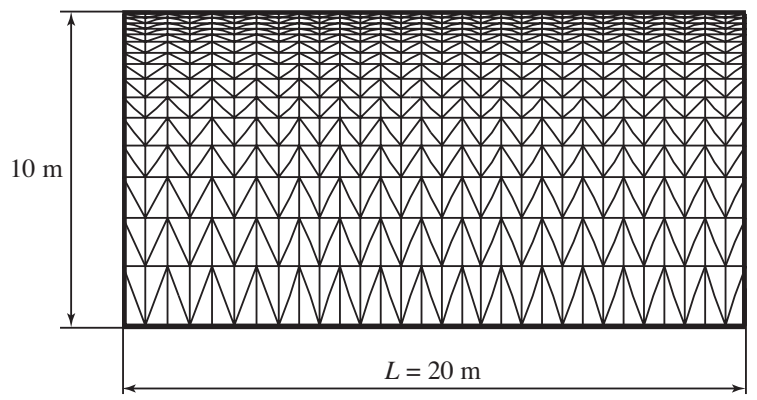

Figure 3: The mesh for the test problem
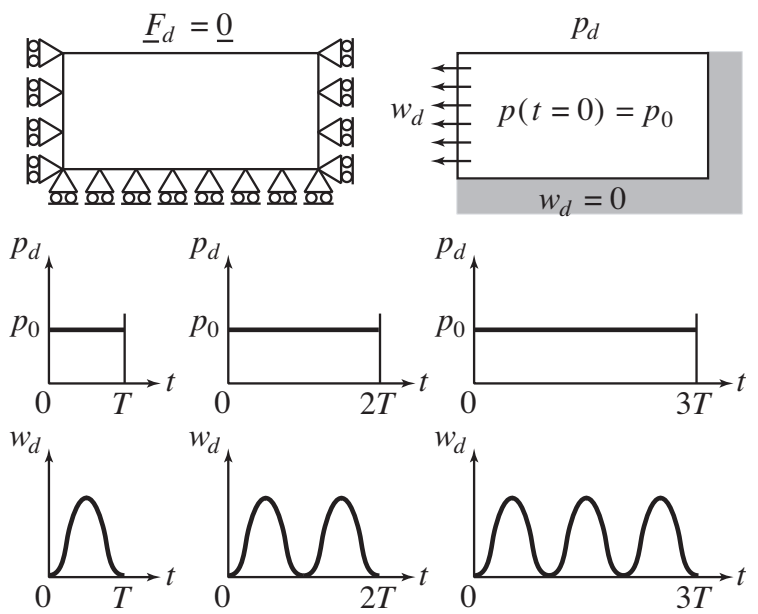

Figure 4: The fluid-flux-driven test problem (top: prescribed solid and fluid quantities; bottom: time evolution of the loading)

\begin{tabular}{lcccc}
\hline & test case & 1 & 2 & 3 \\
& $n_{T}$ & 60 & 120 & 180 \\
\hline \multirow{2}{*}{ ISPP } & $n_{S}$ & 240 & 600 & 900 \\
& $n_{F}$ & 240 & 600 & 900 \\
& $n_{\text {sub }}$ & 4 & 5 & 5 \\
\hline \multirow{2}{*}{ LATIN } & $n_{S}$ & 720 & 1,920 & 3,420 \\
without P3 & $n_{F}$ & 720 & 1,920 & 3,420 \\
& $n_{\text {it }}$ & 12 & 16 & 19 \\
\hline \multirow{2}{*}{ LATIN } & $n_{S}$ & 4 & 6 & 11 \\
with 1/2P3 & $n_{F}$ & 8 & 10 & 12 \\
& $n_{\text {it }}$ & 16 & 20 & 21 \\
\hline
\end{tabular}

Table 3: Number of global resolutions for the fluid-flux-driven problem 
With the ISPP method, convergence was better for the fluid-flux-driven problem than for the force-driven problem: only about half as many subcycles were needed to reach convergence. With the LATIN approach, convergence was almost the same. The interesting point was the behavior of the algorithm when the time complexity of the loading increased. With preserved convergence (i.e. a constant number of subcycles $n_{\text {sub }}$ ), the cost of the ISPP method is directly related to the number of time steps $n_{T}$. For the LATIN method with the radial loading approximation, the number of space fields generated $n_{S}+n_{F}$ increases very slowly with the complexity of the loading path $n_{T}$ and so does the cost of the simulation.

\section{A MULTI-TIME-SCALE APPROACH}

The use of different time scales within the same structural analysis can be accomplished in different ways depending on the nature of the different scales. For instance, recent works in dynamics [CG02] deal with the coupling of different numerical integration schemes in different areas of the structures (substructures): explicit with small time steps, implicit with larger time steps. In weak fluid-structure interaction, coupling is classically introduced through subcycling, usually on the fluid quantities, to satisfy both stability and accuracy requirements, see e.g. [FPF01]. For heterogeneous problems, both scales are encountered at each point of the structure [FW01 YF02] and time homogenization is used to improve the algorithms, as in [LN02], where homogenization is applied to the effective space scale throughout the interfaces between substructures. Another example is found in [MT02], where the solution is corrected iteratively at both time scales in a multigrid-like approach.

For the multiphysics problems we are concerned with, the use of different time scales for the solid and the fluid in the LATIN method does not affect the linear stage, in which all the calculations are conducted independently for each physics. Only the local stage involves coupling of the different physics and, therefore, must deal with the different time scales.

\subsection{The multiscale local stage at iteration $n+1$}

Since the solid and fluid unknowns interact with one another, this subsection presents a strategy to deal with the different time scales. This method is a modified version of the micro-macro strategy proposed in [LD99, LLD01] to solve multi-space-scale problems.

The method consists of splitting the unknowns into $s=s^{M}+s^{m}$, where $s^{M}$ is the set of the "macroscopic" quantities and $s^{m}$ is the "micro" complement. Here, we make the a priori choice to express the state variables of

- the fluid part using both a macro scale and a micro scale: $p=p^{M}+p^{m}$ and $q=q^{M}+q^{m}$;

- the solid part using a macro scale only: $\boldsymbol{\sigma}=\boldsymbol{\sigma}^{M}$ and $\varepsilon=\varepsilon^{M}$

where the superscripts ${ }^{m}$ and ${ }^{M}$ designate the micro and macro time scales respectively. Clearly, the splitting into micro and macro parts is physics-based since we choose to define the macro time scale as the scale suited to describe the evolution of the solid quantities. As we expect the fluid part to require refined time steps (this will be examplified in the following developments), the complementary part of the evolution of the fluid quantities, with respect to the macro time steps, is described herein as the micro time scale.

Using the projector $\boldsymbol{\pi}$, the macro part of a time function $f \in \mathcal{F}_{[0, T]}$ is given by $f^{M}=\boldsymbol{\pi} f \in \mathcal{F}_{[0, T]}^{M}$. Then, the micro part is the complement $f^{m}=(\mathbf{i d}-\boldsymbol{\pi}) f \in \mathcal{F}_{[0, T]}^{m}$ of the macro part. $\mathcal{F}_{[0, T]}^{M}$ and $\mathcal{F}_{[0, T]}^{m}$ designate the spaces of the micro and macro time functions respectively.

Let $\mathbf{e}^{M}=\left\{e_{0}^{M}, \ldots, e_{n_{M}}^{M}\right\}$ be a basis of $\mathcal{F}_{[0, T]}^{M}$ orthonormal with respect to the scalar product $\langle f, g\rangle=$ $\int_{0}^{T} f g d t . \pi$ can be expanded as:

$$
f^{M}=\boldsymbol{\pi} f=\sum_{k=0}^{n_{M}}\left\langle f, e_{k}^{M}\right\rangle e_{k}^{M} \quad \text { and } \quad f^{m}=f-f^{M}
$$

The micro-macro decomposition is performed at the level of the continuum and involves no discretization.

Let us consider a partition of the time interval $[0, T]$ into $n_{M}$ subintervals $\left\{I_{1}, \ldots, I_{n_{M}}\right\}$ given by $I_{k}=$ $\left[T_{k-1}, T_{k}\right]$ with nodes $0=T_{0}<T_{1}<\cdots<T_{n_{M}}=T$. We define, for instance, $\mathcal{F}_{[0, T]}^{M}$ as the space of the functions which are continuous and linear over each $I_{k}$. An orthonormal basis $\mathbf{e}^{M}$ can be constructed using a Schmidt procedure on the $n_{M}+1$ usual basis functions of $\mathcal{F}_{[0, T]}^{M}$. Figure 5 shows an example of a function $f$ with its macro part. Contrary to a hierarchical approach, it is not necessary for $f^{M}\left(T_{k}\right)$ to be equal to $f\left(T_{k}\right)$.

For the multiphysics problem we are dealing with, we define 2 time discretizations:

- for the fluid part, $n$ subintervals $i_{k}=\left[t_{k-1}, t_{k}\right]$ with the nodes $0=t_{0}<\cdots<t_{n}=T$;

- for the solid part, $n_{M}$ subintervals $I_{k}=\left[T_{k-1}, T_{k}\right]$ with the nodes $0=T_{0}<\cdots<T_{n_{M}}=T$.

We choose to set $n_{M} \leqslant n$ and, for simplicity's sake, the time discretization for the solid is assumed to be a subset of that for the fluid, as shown in Figure 6 


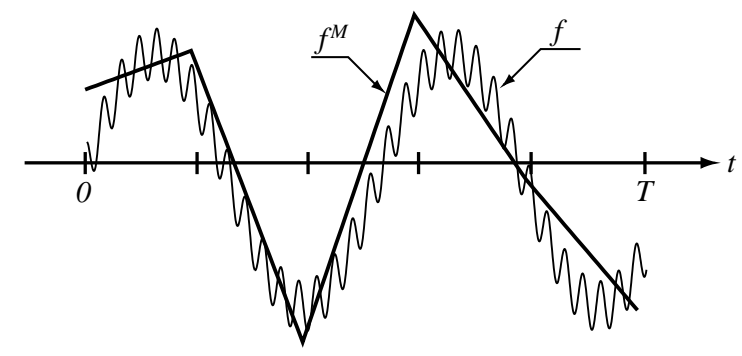

Figure 5: The macro part $f^{M}$ of a function $f$

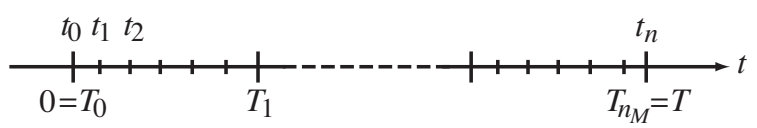

Figure 6: An example of time discretizations for the solid and the fluid

With $\left(\hat{\dot{\varepsilon}}, \hat{\varepsilon}, \mathbf{A}_{n}\right)$ described on the solid mesh (macro scale) and ( $\left.\hat{\dot{p}}, \hat{p}, \alpha_{n}\right)$ on the fluid mesh (both macro and micro scales), the local stage (7) is transformed into:

$$
\begin{gathered}
\mathbf{L} \hat{\dot{\varepsilon}}+\mathbf{D} \hat{\varepsilon}=\mathbf{A}_{n}(t)+b[\boldsymbol{\pi} \hat{p}] \mathbf{1} \\
\frac{1}{Q} \hat{\dot{p}}+r \hat{p}=\alpha_{n}(t)-b \operatorname{Tr}[\mathbf{P} \hat{\dot{\varepsilon}}]
\end{gathered}
$$

where $\boldsymbol{\pi}$ is the previous projector and $\mathbf{P}$ a linear interpolation. $11 \mathrm{a}$ can be solved on the solid's time discretization whereas $11 \mathrm{~b}$ ) can be solved on the fluid's.

Such a local stage is a simplified form of (7) when the fluid part is expected to experience rapid evolution (micro part) which does not influence the solid behavior. Indeed, when the search directions are optimized for the LATIN approach without the multi-time-scale feature, the ordinary differential equation one must solve in (11a) has a characteristic time $t_{m}=80 \mathrm{~ms}$ (so that $\mathbf{L}=t_{m} \mathbf{D}$ ), while the macro time step is $31 \mathrm{~ms}$ and the micro time step varies between $0.49 \mathrm{~ms}$ and $31 \mathrm{~ms}$ for the subsequent numerical simulations. Since the micro time scale is less than $t_{m}$, the previous approximation is expected to be valid.

This new local stage is performed using a fixed point method. The initial guess for the pressure is chosen as $\hat{p}=p_{n}$. Once $\hat{p}$ is known, one can get $\hat{\dot{\varepsilon}}$ using (11a), then $\hat{p}$ using 11b]. The choice of the number of subiterations will be discussed in another section.

\subsection{Numerical results of the multiscale approach}

Let us now consider the force-driven problem of Section 3.2. this time in the one-dimensional case. The time interval is $T=1 \mathrm{~s}$ with $t_{1}=T / 2$ and the pressures are $p_{1}=10 \mathrm{MPa}$ and $p_{0}=0.1 \mathrm{MPa}$; the initial condition is $p(t=0)=p_{0}$; the height of the structure is $L=5 \mathrm{~m}$, discretized into 100 elements. The search direction parameters are set to $t_{m}=910^{-3} t_{c}$ and $t_{h}=8010^{-4} t_{c}$, where $t_{c}=9.3 \mathrm{~s}$. Note that in each of the following numerical tests, the time steps are greater than the critical time step $\Delta t_{\min }=0.17 \mathrm{~ms}$.

The simulation was performed with the LATIN method without P3. The solid part was assumed to have only a macro time scale with $n_{M}=32$ time steps and the fluid part was discretized with $n$ time steps. The influence of $n$ on the fluid's contribution to the global error was tested. As before, this error was computed with respect to a reference solution $\mathbf{s}_{e x}$ obtained with the direct monolithic approach and a very fine time discretization; the time step used was $\Delta t=0.24 \mathrm{~ms}>\Delta t_{\min }=0.17 \mathrm{~ms}$. In such a case, the solution no longer converges toward the reference solution and the value of the error stabilizes as the algorithms converge. In this particular instance, the time step ratio was $\Delta t_{S} / \Delta t_{F}=n / n_{M}$.

Table 4 shows the evolution of the contributions $\eta_{S}$ and $\eta_{F}$ to the total error $\eta$ against $\Delta t_{S} / \Delta t_{F}$. Since we are dealing with a coupled problem, a refinement of the time discretization for the fluid decreases both contributions. However, the fluid's contribution decreases more than the solid's. When the description of the fluid part is sufficiently refined, it can be considered continuous in time. The corresponding contributions are $\eta_{S}^{\infty}$ and $\eta_{F}^{\infty}$, neither of which is zero since the coarse discretization of the solid part affects both errors. Figure 77a) shows the evolution of $\eta_{F}^{2} / \eta_{S}^{\infty}$ with $\Delta t_{S} / \Delta t_{F}$

To study the error in the fluid part due to the fluid discretization alone, let us consider the quantity $\eta_{F}^{2}-\eta_{F}^{\infty}$. With the numerical convergence of $\eta_{F}^{2}-\eta_{F}^{\infty}$ obtained as shown in Figure $7 \mathrm{~b}$ ), one can observe that in order to get similar contributions to the error from the fluid part and from the solid part the time step ratio must be $\Delta t_{S} / \Delta t_{F} \sim 16$. 


\begin{tabular}{ccccc}
\hline$\frac{\Delta t_{S}}{\Delta t_{F}}$ & $\eta_{S}$ & $\eta_{F}$ & $\eta$ & $\frac{\eta_{F}}{\eta_{S}}$ \\
\hline 1 & $12.910^{-4}$ & $17.910^{-4}$ & $22.110^{-4}$ & 1.39 \\
2 & $6.4710^{-4}$ & $8.9710^{-4}$ & $11.110^{-4}$ & 1.38 \\
4 & $3.2710^{-4}$ & $4.3310^{-4}$ & $5.5210^{-4}$ & 1.35 \\
8 & $1.7210^{-4}$ & $2.1810^{-4}$ & $2.7810^{-4}$ & 1.26 \\
16 & $1.0510^{-4}$ & $1.1010^{-4}$ & $1.5210^{-4}$ & 1.05 \\
32 & $0.8210^{-4}$ & $0.7110^{-4}$ & $1.0810^{-4}$ & 0.86 \\
64 & $0.7710^{-4}$ & $0.6610^{-4}$ & $1.0110^{-4}$ & 0.85 \\
\multicolumn{5}{c}{$=\eta_{S}^{\infty}$} \\
\hline
\end{tabular}

Table 4: Error contributions when the solid part is assumed to be macro and $\Delta t_{S}=T / 32$

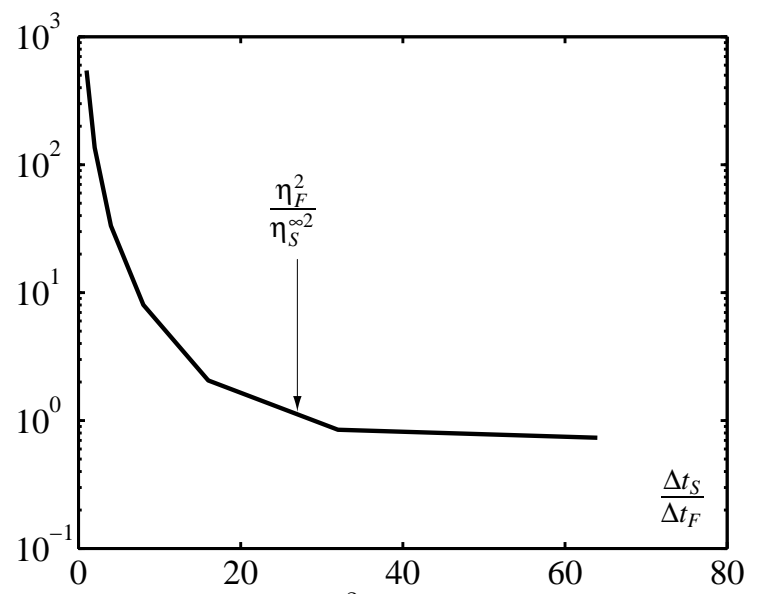

(a) $\frac{\eta_{F}^{2}}{\eta_{S}^{\infty}}$ vs. $\frac{\Delta t_{S}}{\Delta t_{F}}$

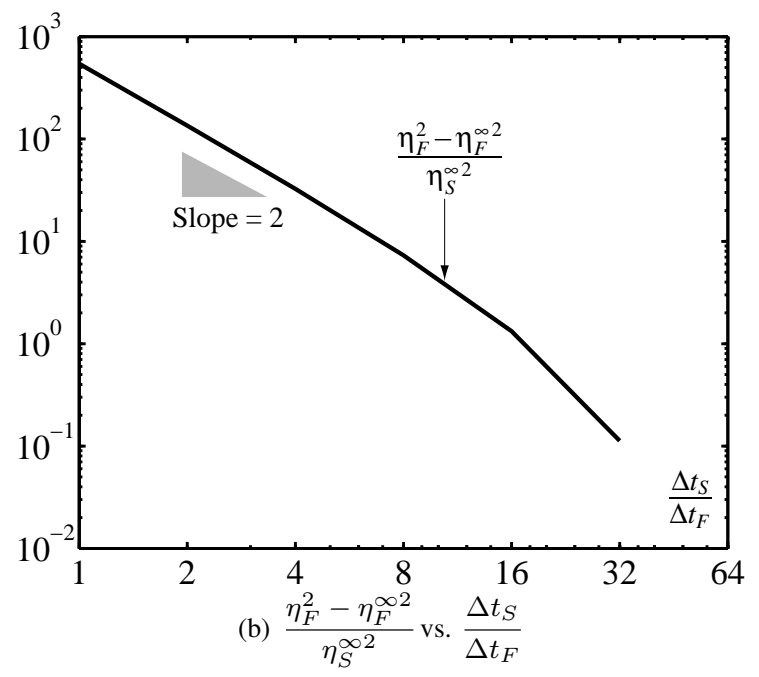

Figure 7: Error contributions with the multi-time-scale strategy

Figure 8 shows the influence of the number of subiterations in the local stage on the total error $\eta$ when the time discretizations consist of 32 time steps for the solid and 64 for the fluid. The convergence of the local stage took at least 2 subiterations and this number had little influence on the approach. In all subsequent tests, 2 subiterations were used for the local stage.

As the numerical tests about the multi-time-scale approach are up to now only implemented for 1D problems, both the force-driven problem and fluid-flux driven are very similar, and are expected to lead to the same behavior. 


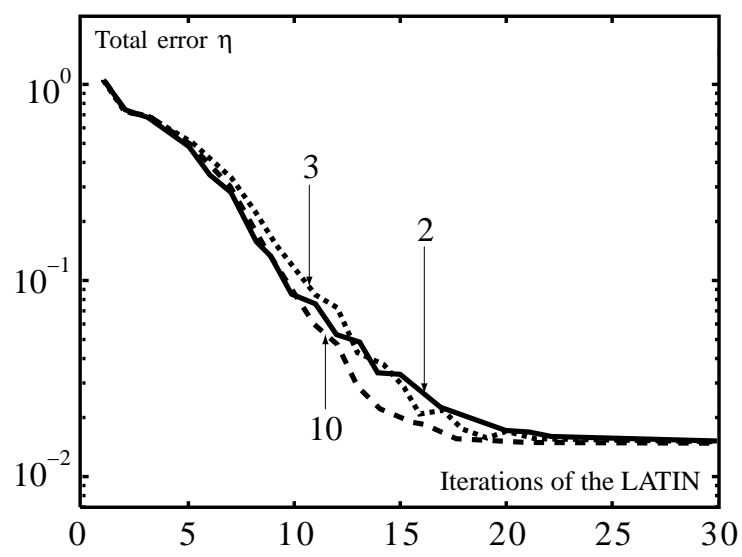

Figure 8: Influence of the number of subiterations in the local stage

\section{THE RADIAL LOADING APPROXIMATION P3}

In the previous paper [DLS03], the last principle of the LATIN approach (i.e. a suitable approximation of the unknowns) was necessary to achieve good computational efficiency.

Classically, this consists of expressing the correction $\Delta \mathbf{s}=\mathbf{s}_{n+1}-\mathbf{s}_{n}$ on a radial loading basis; in other terms, the corrections to the unknowns are sought in the form of the product of a space field by a scalar time function.

In the previous paper, the approximation was carried out only for the kinematic field corrections $\Delta \dot{\varepsilon}$ and $\Delta p$, leading to the so-called $1 / 2 \mathrm{P} 3$ version. The advantage was that the global systems to be solved were smaller (see previous sections), but there was no gain in storage costs because, as for the LATIN method without the radial loading approximation, one had to store the values of the unknowns at each Gauss point and for each time step.

In this paper, we are investigating the option of approximating both the kinematic and the static quantities, which we refer to as the P3 version. Provided this option does not decrease the convergence rate, it results in a reduction of the storage cost also because it requires only a few space fields and scalar time functions (the number generated by the algorithm).

\subsection{The linear stage with full approximation (P3) at iteration $n+1$}

Let us rewrite the search direction $(8)$ in terms of the correction with respect to $\mathbf{s}_{n}$ :

$$
\begin{aligned}
& \Delta \boldsymbol{\sigma}-\mathbf{L} \Delta \dot{\boldsymbol{\varepsilon}}+\Delta \hat{\mathbf{A}}=0 \\
& \Delta \underline{W}-\mathbf{H} \Delta \underline{Z}+\Delta \underline{\hat{W}}=0 \\
& \Delta q-r \Delta p+\Delta \hat{\alpha}=0
\end{aligned}
$$

where $\Delta \dot{\varepsilon}=\dot{\varepsilon}_{n+1}-\dot{\varepsilon}_{n}, \Delta \boldsymbol{\sigma}=\boldsymbol{\sigma}_{n+1}-\boldsymbol{\sigma}_{n} \ldots$ are the corrections and:

$$
\begin{array}{r}
\Delta \hat{\mathbf{A}}=(\mathbf{L} \hat{\dot{\varepsilon}}-\hat{\boldsymbol{\sigma}})-\left(\mathbf{L} \dot{\boldsymbol{\varepsilon}}_{n}-\boldsymbol{\sigma}_{n}\right) \\
\Delta \underline{\hat{W}}=(\mathbf{H} \underline{\hat{Z}}-\underline{\hat{W}})-\left(\mathbf{H} \underline{Z}_{n}-\underline{W}_{n}\right) \\
\Delta \hat{\alpha}=(r \hat{p}-\hat{q})-\left(r p_{n}-q_{n}\right)
\end{array}
$$

At this stage, $\Delta \hat{\mathbf{A}}, \Delta \underline{\hat{W}}$ and $\Delta \hat{\alpha}$ are known quantities.

One has to find $\Delta \mathbf{s} \in \mathbf{A}_{\mathbf{d} 0}$ which follows the search direction (12) best, i.e. which minimizes the following errors in constitutive relation [LP01]:

$$
\frac{1}{2} \int_{0}^{T} \int_{\Omega} \operatorname{Tr}\left[(\Delta \boldsymbol{\sigma}-\mathbf{L} \Delta \dot{\boldsymbol{\varepsilon}}+\Delta \hat{\mathbf{A}}) \mathbf{L}^{-1}(\Delta \boldsymbol{\sigma}-\mathbf{L} \Delta \dot{\boldsymbol{\varepsilon}}+\Delta \hat{\mathbf{A}})\right] d \Omega d t
$$

and

$$
\begin{aligned}
\frac{1}{2} \int_{0}^{T} \int_{\Omega}\left\{(\Delta q-r \Delta p+\Delta \hat{\alpha}) r^{-1}(\Delta q\right. & -r \Delta p+\Delta \hat{\alpha}) \\
+ & \left.(\Delta \underline{W}-\mathbf{H} \Delta \underline{Z}+\Delta \underline{\hat{W}}) \cdot \mathbf{H}^{-1}(\Delta \underline{W}-\mathbf{H} \Delta \underline{Z}+\Delta \underline{\hat{W}})\right\} d \Omega d t
\end{aligned}
$$




\subsubsection{Correction for the solid part}

The previous minimization problem leads to the kinematic correction $\Delta \dot{\varepsilon} \in \mathcal{E}_{0}^{[0, T]}$ such that:

$$
\forall \dot{\varepsilon}^{\star} \in \mathcal{E}_{0}^{[0, T]},
$$

$$
\int_{0}^{T} \int_{\Omega}\left\{\operatorname{Tr}\left[\Delta \dot{\varepsilon} \mathbf{L} \dot{\varepsilon}^{\star}\right]-\operatorname{Tr}\left[\Delta \hat{\mathbf{A}} \dot{\varepsilon}^{\star}\right]\right\} d \Omega d t=0
$$

and the static correction $\Delta \sigma \in \mathcal{S}_{0}^{[0, T]}$ such that:

$$
\forall \boldsymbol{\sigma}^{\star} \in \mathcal{S}_{0}^{[0, T]},
$$

$$
\int_{0}^{T} \int_{\Omega}\left\{\operatorname{Tr}\left[\Delta \boldsymbol{\sigma} \mathbf{L}^{-1} \boldsymbol{\sigma}^{\star}\right]+\operatorname{Tr}\left[\Delta \hat{\mathbf{A}} \mathbf{L}^{-1} \boldsymbol{\sigma}^{\star}\right]\right\} d \Omega d t=0
$$

The kinematic correction is represented in the approximate form $\Delta \dot{\varepsilon}=v \varepsilon(\underline{V})$, where $v(t)$ is a scalar time function defined on the solid time grid and the space field $\underline{V}(M) \in \mathcal{U}_{0}$. In order to determine the pair $(v, \underline{V})$ uniquely, normalization is enforced: $\int_{\Omega} \operatorname{Tr}[\varepsilon(\underline{V}) \mathbf{L} \varepsilon(\underline{V})] d \Omega=1$. Then, the pair is such that:

$$
v(t)=\int_{\Omega} \operatorname{Tr}[\Delta \hat{\mathbf{A}}(t) \varepsilon(\underline{V})] d \Omega
$$

and

$$
\forall \underline{V}^{\star} \in \mathcal{U}_{0}, \int_{\Omega} \operatorname{Tr}\left[\varepsilon(\underline{V}) \mathbf{L} \varepsilon\left(\underline{V}^{\star}\right)\right] d \Omega
$$

$$
=\int_{0}^{T} \frac{v}{\|v\|_{t}^{2}} \int_{\Omega} \operatorname{Tr}\left[\Delta \hat{\mathbf{A}} \varepsilon\left(\underline{V}^{\star}\right)\right] d \Omega d t
$$

where $\|v\|_{t}^{2}=\int_{0}^{T} v^{2} d t$.

This coupled system of equations can be solved using a fixed-point method between $v$ and $\underline{V}$ and the number of subiterations is usually small (in practice, for the following tests, we used only one subiteration).

The static correction is also represented in the approximate form $\Delta \boldsymbol{\sigma}=s(t) \boldsymbol{S}(M)$, where $s(t)$ is a scalar time function defined on the solid time grid and the space field $\boldsymbol{S}(M) \in \mathcal{S}_{0}$. The pair $(s, \boldsymbol{S})$, normalized by $\int_{\Omega} \operatorname{Tr}\left[\boldsymbol{S} \mathbf{L}^{-1} \boldsymbol{S}\right] d \Omega=1$, is such that:

$$
s(t)=-\int_{\Omega} \operatorname{Tr}\left[\Delta \hat{\mathbf{A}}(t) \mathbf{L}^{-1} \boldsymbol{S}\right] d \Omega
$$

and

$$
\begin{aligned}
\forall \boldsymbol{S}^{\star} \in \mathcal{S}_{0}, \quad \int_{\Omega} \operatorname{Tr}\left[\boldsymbol{S} \mathbf{L}^{-1} \boldsymbol{S}^{\star}\right] d \Omega & \\
& =-\int_{0}^{T} \frac{s}{\|s\|_{t}^{2}} \int_{\Omega} \operatorname{Tr}\left[\Delta \hat{\mathbf{A}} \mathbf{L}^{-1} \boldsymbol{S}^{\star}\right] d \Omega d t
\end{aligned}
$$

This coupled system of equations can be solved with the same fixed-point method (with, in practice, only one subiteration).

Since it is not easy, in a displacement-oriented finite element code, to search for $\boldsymbol{S}(M) \in \mathcal{S}_{0}$ (statically admissible fields), the last equation is classically solved by a dualization procedure: that condition is replaced by the equivalent one which consists of forcing $\mathbf{L}^{-1} \boldsymbol{S}+\hat{\boldsymbol{T}}$ to be kinematically admissible to zero, where:

$$
\hat{\boldsymbol{T}}=\int_{0}^{T} \frac{s}{\|s\|_{t}^{2}} \mathbf{L}^{-1} \Delta \hat{\mathbf{A}} d t
$$

i.e. to be of the form $\mathbf{L}^{-1} \boldsymbol{S}+\hat{\boldsymbol{T}}=\varepsilon(\underline{X})$ with $\underline{X} \in \mathcal{U}_{0}$. Since $\boldsymbol{S}=\mathbf{L} \varepsilon(\underline{X})-\mathbf{L} \hat{\boldsymbol{T}} \in \mathcal{S}_{0}$, the dual problem consists of finding $\underline{X} \in \mathcal{U}_{0}$ such that:

$$
\forall \underline{X}^{\star} \in \mathcal{U}_{0}, \quad \int_{\Omega} \operatorname{Tr}\left[\varepsilon(\underline{X}) \mathbf{L} \varepsilon\left(\underline{X}^{\star}\right)\right] d \Omega
$$

$$
=\int_{\Omega} \operatorname{Tr}\left[\hat{\boldsymbol{T}} \mathbf{L} \varepsilon\left(\underline{X}^{\star}\right)\right] d \Omega d t
$$

Once $\underline{X}$ has been calculated, $\boldsymbol{S}=\mathbf{L} \varepsilon(\underline{X})-\mathbf{L} \hat{\boldsymbol{T}}$.

Let us note that if the kinematic correction is calculated first, the fixed-point method can be initialized with $(s, \underline{X})=(v, \underline{V})$. 


\subsubsection{Correction for the fluid part}

The previous minimization leads to seeking the kinematic corrections $\Delta p \in \mathcal{P}_{0}^{[0, T]}$ and $\Delta \underline{Z}=\operatorname{grad} \Delta p$ such that:

$$
\forall p^{\star} \in \mathcal{P}_{0}^{[0, T]}, \quad \int_{0}^{T} \int_{\Omega}\left\{\Delta p r p^{\star}+\Delta \underline{Z} \cdot \mathbf{H} \underline{\operatorname{grad}} p^{\star}\right.
$$

$$
\left.-\Delta \hat{\alpha} p^{\star}-\Delta \underline{\hat{W}} \cdot \underline{\operatorname{grad}} p^{\star}\right\} d \Omega d t=0
$$

and the static corrections $(\Delta q, \Delta \underline{W}) \in \mathcal{H}_{0}^{[0, T]}$ such that:

$$
\begin{aligned}
& \forall\left(q^{\star}, \underline{W^{\star}}\right) \in \mathcal{H}_{0}^{[0, T]}, \quad \int_{0}^{T} \int_{\Omega}\left\{\Delta q r^{-1} q^{\star}+\Delta \underline{W} \cdot \mathbf{H}^{-1} \underline{W^{\star}}\right. \\
&\left.+\Delta \hat{\alpha} r^{-1} q^{\star}+\Delta \underline{\hat{W}} \cdot \mathbf{H}^{-1} \underline{W^{\star}}\right\} d \Omega d t=0
\end{aligned}
$$

The kinematic correction is represented in the approximate form $\Delta p=\pi P$ and $\Delta \underline{Z}=\pi \operatorname{grad} P$, where $\pi(t)$ is a scalar time function defined on the fluid time grid and the space field $P(M) \in \mathcal{P}_{0}$. The pair $(\pi, P)$, normalized by $\int_{\Omega}(\operatorname{Pr} P+\underline{\operatorname{grad}} P \cdot \mathbf{H} \underline{\operatorname{grad}} P) d \Omega=1$, is such that:

$$
\begin{gathered}
\pi=\int_{\Omega}(\Delta \hat{\hat{\alpha}} P+\Delta \underline{\hat{W}} \cdot \underline{\operatorname{grad}} P) d \Omega \\
\forall P^{\star} \in \mathcal{P}_{0}, \quad \int_{\Omega}\left(P r P^{\star}+\underline{\operatorname{grad}} P \cdot \underline{\mathbf{H}} \underline{\operatorname{grad}} P^{\star}\right) d \Omega \\
\quad=\int_{0}^{T} \frac{\pi}{\|\pi\|_{t}^{2}} \int_{\Omega}\left(\Delta \hat{\alpha} P^{\star}+\Delta \underline{\hat{W}} \cdot \underline{\operatorname{grad}} P^{\star}\right) d \Omega d t
\end{gathered}
$$

This coupled system of equations can be solved by the same fixed-point method as before using only a small number of subiterations (in the following tests, we also used one subiteration).

The static correction is sought in the approximate form $\Delta q=\theta(t) Q(M)$ and $\Delta \underline{W}=\theta(t) \underline{Y}(M)$, where $\theta(t)$ is a scalar time function defined on the fluid time grid and the space fields $(Q, \underline{Y}) \in \mathcal{H}_{0}$. The triplet $(\theta, Q, \underline{Y})$, normalized by $\int_{\Omega}\left(Q r^{-1} Q+\underline{Y} \cdot \mathbf{H}^{-1} \underline{Y}\right) d \Omega=1$, is such that:

$$
\theta=-\int_{\Omega}\left(\Delta \hat{\alpha} r^{-1} Q+\Delta \underline{\hat{W}} \cdot \mathbf{H}^{-1} \underline{Y}\right) d \Omega
$$

and

$$
\begin{aligned}
\forall\left(Q^{\star}, \underline{Y}^{\star}\right) \in \mathcal{H}_{0}, \quad \int_{\Omega}\left(Q r^{-1} Q^{\star}+\underline{Y} \cdot \mathbf{H}^{-1} \underline{Y}^{\star}\right) d \Omega & \\
& =-\int_{0}^{T} \frac{\theta}{\|\theta\|_{t}^{2}} \int_{\Omega}\left(\Delta \hat{\alpha} r^{-1} Q^{\star}+\Delta \underline{\hat{W}} \cdot \mathbf{H}^{-1} \underline{Y}^{\star}\right) d \Omega d t
\end{aligned}
$$

As in the case of the solid part, once $\theta$ is known, the last equation is classically solved by dualization with:

$$
\begin{aligned}
& Q=r \Pi-\int_{0}^{T} \frac{\theta}{\|\theta\|_{t}^{2}} \Delta \hat{\alpha} d t \\
& \underline{Y}=\mathbf{H} \underline{\operatorname{grad}} \Pi-\int_{0}^{T} \frac{\theta}{\|\theta\|_{t}^{2}} \Delta \underline{\hat{W}} d t
\end{aligned}
$$

where $\Pi \in \mathcal{P}_{0}$ is statically admissible to zero. The dual problem consists of finding $\Pi \in \mathcal{P}_{0}$ such that:

$$
\begin{aligned}
\forall P^{\star} \in \mathcal{P}_{0}, \quad \int_{\Omega}\left(\Pi r P^{\star}+\underline{\operatorname{grad}} \Pi \cdot \mathbf{H} \underline{\operatorname{grad}} P^{\star}\right) d \Omega & \\
& =\int_{0}^{T} \frac{\theta}{\|\theta\|_{t}^{2}} \int_{\Omega}\left(\Delta \hat{\alpha} P^{\star}+\Delta \underline{\hat{W}} \cdot \underline{\operatorname{grad}} P^{\star}\right) d \Omega d t
\end{aligned}
$$

As in the case of the solid part, the fixed-point method can be initialized with $(\theta, \Pi)=(\pi, P)$.

\subsection{The preliminary stage at iteration $n+1$}

Once several space fields have been generated in previous iterations, the objective is to update the time functions of the various approximations and reuse the previously built space fields before a possible linear stage [Lad99]. This stage is much simpler if the space fields are orthogonalized each time a new space field is added to the existing set. 


\subsubsection{Preliminary stage for the solid part}

This time, one uses a correction of the form $\Delta \dot{\varepsilon}=\sum_{j} w_{j} \varepsilon\left(\underline{V}_{j}\right)$ and $\Delta \boldsymbol{\sigma}=\sum_{j} t_{j} \boldsymbol{S}_{j}$, where $w_{j}$ and $t_{j}$ are the time functions to be determined, but $\underline{V}_{j}$ and $\boldsymbol{S}_{j}$ are known. Thus, the new fields are:

$$
\begin{aligned}
& \overline{\dot{\varepsilon}}_{n+1}=v_{0} \varepsilon\left(\underline{V}_{0}\right)+\sum_{j}\left(v_{j}+w_{j}\right) \varepsilon\left(\underline{V}_{j}\right) \\
& \overline{\boldsymbol{\sigma}}_{n+1}=s_{0} \boldsymbol{S}_{0}+\sum_{j}\left(s_{j}+t_{j}\right) \boldsymbol{S}_{j}
\end{aligned}
$$

Searching for $w_{j}$ and $t_{j}$ is very similar to going through the first step of the previous fixed-point methods during the linear stages. If the space fields are orthonormal, i.e.

$$
\int_{\Omega} \operatorname{Tr}\left[V_{i} \mathbf{L} V_{j}\right] d \Omega=\delta_{i j} \quad \text { and } \quad \int_{\Omega} \operatorname{Tr}\left[\boldsymbol{S}_{i} \mathbf{L}^{-1} \boldsymbol{S}_{j}\right] d \Omega=\delta_{i j}
$$

this leads to:

$$
\begin{aligned}
w_{j} & =\int_{\Omega} \operatorname{Tr}\left[\varepsilon\left(\underline{V}_{j}\right) \Delta \hat{A}\right] \\
t_{j} & =-\int_{\Omega} \operatorname{Tr}\left[\boldsymbol{S}_{j} \mathbf{L}^{-1} \Delta \hat{A}\right]
\end{aligned}
$$

\subsubsection{Preliminary stage for the fluid part}

This time, one uses a correction of the form $\Delta p=\sum_{j} \varpi_{j} P_{j}, \Delta \underline{Z}=\sum_{j} \varpi_{j} \operatorname{grad} P_{j}$ and $\Delta q=\sum_{j} \vartheta_{j} Q_{j}$, $\Delta \underline{W}=\sum_{j} \vartheta_{j} \underline{Y}_{j}$, where $\varpi_{j}$ and $\vartheta_{j}$ are the time functions to be determined, but $P_{j}, Q_{j}$ and $\underline{Y}_{j}$ are known. Thus, the new fields are:

$$
\begin{aligned}
\bar{p}_{n+1} & =\pi_{0} P_{0}+\sum_{j}\left(\pi_{j}+\varpi_{j}\right) P_{j} \\
\underline{\bar{Z}}_{n+1} & =\pi_{0} \underline{\operatorname{grad}} P_{0}+\sum_{j}\left(\pi_{j}+\varpi_{j}\right) \underline{\operatorname{grad}} P_{j}
\end{aligned}
$$

and

$$
\begin{aligned}
\bar{q}_{n+1} & =\theta_{0} Q_{0}+\sum_{j}\left(\theta_{j}+\vartheta j\right) Q_{j} \\
\underline{\bar{W}}_{n+1} & =\theta_{0} \underline{Y}_{0}+\sum_{j}\left(\theta_{j}+\vartheta j\right) \underline{Y}_{j}
\end{aligned}
$$

If the space fields are orthonormal, i.e.

$$
\begin{aligned}
\int_{\Omega}\left(P_{i} r P_{j}+\underline{\operatorname{grad}} P_{i} \cdot \mathbf{H} \underline{\operatorname{grad}} P_{j}\right) d \Omega & =\delta_{i j} \\
\int_{\Omega}\left(Q_{i} r^{-1} Q_{j}+\underline{Y}_{i} \cdot \mathbf{H}^{-1} \underline{Y}_{j}\right) d \Omega & =\delta_{i j}
\end{aligned}
$$

this leads to:

$$
\begin{aligned}
\varpi_{j} & =\int_{\Omega}\left(P_{j} \Delta \hat{\alpha}+\underline{\operatorname{grad}} P_{j} \cdot \Delta \underline{\hat{W}}\right) d \Omega \\
\vartheta_{j} & =-\int_{\Omega}\left(Q_{j} r^{-1} \Delta \hat{\alpha}+\underline{Y}_{j} \cdot \mathbf{H}^{-1} \Delta \underline{\hat{W}}\right) d \Omega
\end{aligned}
$$

Once the preliminary stage has been performed, the same strategy as in [DLS03] is used to decide whether new space fields are required to improve accuracy. If not, no linear stage is performed and the next step is the next local stage.

\subsection{Influence of the radial loading approximation}

Let us consider again the force-driven problem of Section 3.2. still in the one-dimensional case. The number of time steps was set to $n_{M}=32$ for the discretization of the solid and to $n=16 n_{M}=512$ for the discretization of the fluid. Figure 9 shows the influence of the approximation on the convergence of the method.

A first calculation was performed without P3. A second calculation was performed with only the kinematic fields being represented (the so-called 1/2P3 used in [DLS03]). Finally, a third calculation was performed with all quantities represented (the so-called P3 described above). 
For the LATIN method with $1 / 2 \mathrm{P} 3$ and $\mathrm{P} 3$, we performed one solid linear stage and one fluid linear stage beyond the preliminary stage, because an accuracy control strategy has not been implemented yet. The number of fixed-point subiterations for each linear stage was set to 1: only one global mechanical problem and one global hydraulic problem were solved at each iteration in the $1 / 2 \mathrm{P} 3$ case and two in the $\mathrm{P} 3$ case. The same search direction parameters as for the previous test were used for this test case.

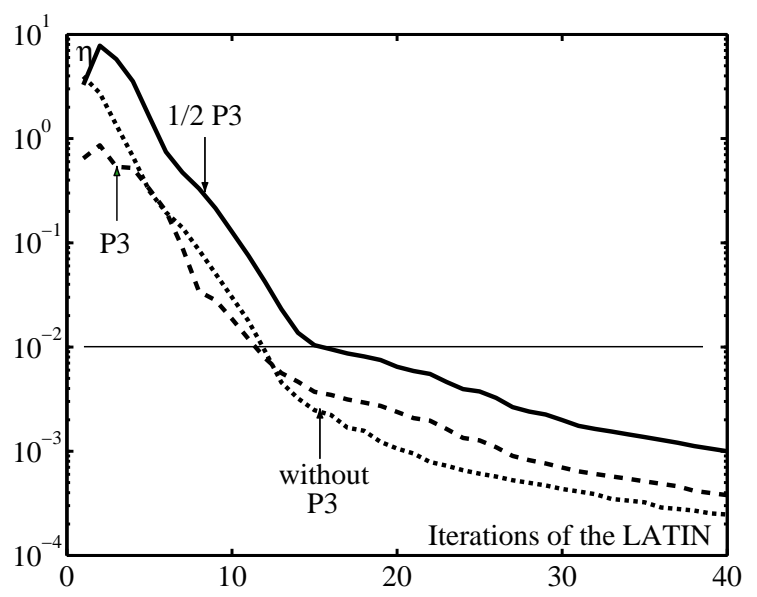

Figure 9: Convergence of the LATIN method with or without representation

The use of a radial loading approximation does not decrease the convergence rate: thus, this is a suitable approximation for this kind of problem. Table 5 illustrates the influence of the third principle of the method on the cost. It gives the number of iterations $n_{\mathrm{it}}$ and the number of global resolutions for the solid $n_{S}$ and for the fluid $n_{F}$ which are necessary to reach a total error $\eta=1 \%$. This error is still evaluated with respect to a reference solution $\mathbf{s}_{e x}$ obtained with the direct monolithic approach and a very fine time discretization.

\begin{tabular}{ccc}
\hline \multirow{2}{*}{ LATIN without P3 } & $n_{S}$ & 384 \\
& $n_{F}$ & 6,144 \\
& $n_{\text {it }}$ & 12 \\
\hline \multirow{2}{*}{ LATIN with 1/2P3 } & $n_{S}$ & 16 \\
& $n_{F}$ & 16 \\
& $n_{\text {it }}$ & 16 \\
\hline \multirow{2}{*}{ LATIN with P3 } & $n_{S}$ & 24 \\
& $n_{F}$ & 24 \\
& $n_{\text {it }}$ & 12 \\
\hline
\end{tabular}

Table 5: Comparative costs of the LATIN method with and without P3

In terms of the number of global resolutions, there is a ratio of 200 between the method without P3 and the $1 / 2 \mathrm{P} 3$ version and a ratio of 140 between the method without $\mathrm{P} 3$ and the $\mathrm{P} 3$ version.

However, the P3 version is the most interesting from the point of view of modularity: in the case of coupling between different FE codes (one for each physical problem), such a representation of all quantities reduces the amount of information to be exchanged considerably. Figure 10 shows a possible flow of information between the two codes. Assuming that the local stages are carried out in each code and that the database is duplicated or shared, the codes are required to exchange one pair of scalar time function and space field only each time a new one is generated, as opposed to at each time step as in a classical incremental approach [FPF01].

\section{REMARK: NONLINEAR PROBLEMS}

In the case of geometrically nonlinear problems [GS01], it is usual to set up an eulerian approach for the fluid and a lagrangian one for the solid. To extend the present strategy to this framework, we have in mind to use the true and new lagrangian approach with corotational description which has been described in [Lad99]. Such a formulation, which is also a rate formulation, can be seen as a common framework for both eulerian and lagrangian descriptions. This approach, well suited to the LATIN method, has been successfully applied for post-buckling and elastomer large transformation problems [BLPR97, ABLM02]. 


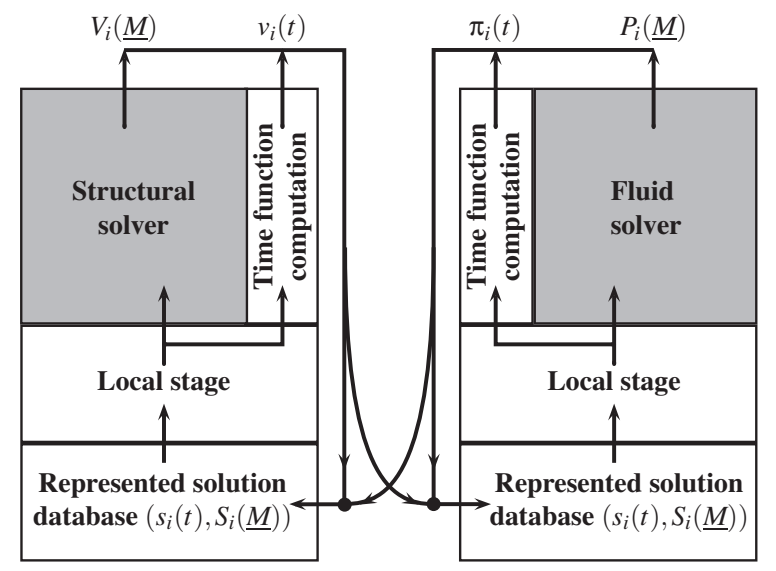

Figure 10: The coupling of FE codes

\section{CONCLUSION}

In this paper, we described a partitioned strategy based on the LATIN approach which enables one to use different time steps for the solid and the fluid parts of a consolidation problem. In particular, a smaller time step is required for the fluid than for the solid in order to perform an iso-quality simulation (i.e. with the same contributions to the global error). In the case of coupling of FE codes, this approach makes it possible for each code to use its own time discretization.

In the numerical tests, uniform time discretization was used. An interesting feature would be for the time discretization to be automatically adaptive. Further work on the use of a time-discontinuous Galerkin formulation is in progress.

The next step will be to expand this technique using the LATIN method to take into account the different space scales which usually arise from the different physics. For problems involving only the solid part, a mixed domain decomposition method based on a micro-macro multiscale computational strategy was shown to be efficient [LLD01]. Such an approach could be easily applied to both the solid and the fluid independently. Moreover, different space discretizations could be used for each physics.

To improve the efficiency of this approach, the third principle of the method must be used. The first tests with the multi-time-scale strategy showed that the convergence rate is the same with or without P3. The radial loading approximation of all state variables improves the efficiency of the approach significantly, especially from the modularity point of view.

Finally, the method has to be tested on other coupled problems, such as thermo-poro-elasticity, which introduce nonlinearities.

\section{References}

[ABLM02] X. Aubard, P.-A. Boucard, P. Ladevèze, and S. Michel. Modeling and simulation of damage in elastomer at high strains. Computers and Structures, 80/27-30:2289-2298, 2002.

[BF91] F. Brezzi and M. Fortin. Mixed and Hybrid Finite Element Methods, volume 15 of Computational Mathematics. Springer, 1991.

[BLL00] P. A. Boucard, P. Ladevèze, and H. Lemoussu. A modular approach to 3-D impact computation with frictional contact. Computer and Structures, 78/1-3:45-52, 2000.

[BLPR97] P.-A. Boucard, P. Ladevèze, M. Poss, and P. Rougée. A nonincremental approach for large displacement problems. Computers and Structures, 64/1-4:499-508, 1997.

[CG02] A. Combescure and A. Gravouil. A numerical scheme to couple subdomains with different timesteps for predominantly linear transient analysis. Computer Methods in Applied Mechanics and Engineering, 191(11-12)(11-12):1129-1157, 2002.

[DL98] D. Dureisseix and P. Ladevèze. A multi-level and mixed domain decomposition approach for structural analysis, volume 218 of Contemporary Mathematics, Domain Decomposition Methods 10, pages 246-253. AMS, 1998

[DLS03] D. Dureisseix, P. Ladevèze, and B. A. Schrefler. A computational strategy for multiphysics problems - application to poroelasticity. International Journal for Numerical Methods in Engineering, 56(10):1489-1510, 2003. 
[FG88] C. A. Felippa and T. L. Geers. Partitioned analysis for coupled mechanical systems. Engineering Computation, 5:123-133, 1988.

[FL00] C. Farhat and M. Lesoinne. Two efficient staggered algorithms for the serial and parallel solution of three-dimensional nonlinear transient aeroelastic poblems. Computer Methods in Applied Mechanics and Engineering, 182:499-515, 2000.

[FP80] C. A. Felippa and K. C. Park. Staggered transient analysis procedures for coupled mechanical systems: formulation. Computer Methods in Applied Mechanics and Engineering, 24:61-111, 1980.

[FPF01] C. A. Felippa, K. C. Park, and C. Farhat. Partitioned analasys of coupled mechanical systems. Computer Methods in Applied Mechanics and Engineering, 190:3247-3270, 2001.

[FW01] J. Fish and W.Chen. Uniformly valid multiple spatial-temporal scale modeling for wave propagation in heterogeneous media. Mechanics of Composite Materials and Structures, 8:81-99, 2001.

[GRE90] GRECO. Scientific report, GRECO Géomatériaux, 1990.

[GS01] W. G. Gray and B. A. Schrefler. Thermodynamic approach to effective stress in partially saturated porous media. Eur. J. Mech. A/Solids, 20:521-538, 2001.

[HKS96] Hibbitt, Karlson, and Sorensen, editors. Abaqus/Standard - User's Manual, volume I, pages 6.4.2-2 and 6.6.1-4. 1996.

[Lad99] P. Ladevèze. Nonlinear Computational Structural Mechanics - New Approaches and NonIncremental Methods of Calculation. Springer Verlag, 1999.

[LBL02] H. Lemoussu, P. A. Boucard, and P. Ladevèze. A 3-D shock computational strategy for real assembly and shock attenuator. Advances in Engineering Software, 33/7-10:517-526, 2002.

[LD99] P. Ladevèze and D. Dureisseix. Une nouvelle stratégie de calcul micro/macro en mécanique des structures. Comptes Rendus à l'Académie des Sciences - Mécanique des Solides et des Structures, (327):1237-1244, 1999.

[LD00] P. Ladevèze and D. Dureisseix. A micro/macro approach for parallel computing of heterogeneous structures. International Journal for Computational Civil and Structural Engineering, 1:18-28, 2000.

[LLD01] P. Ladevèze, O. Loiseau, and D. Dureisseix. A micro-macro and parallel computational strategy for highly heterogeneous structures. International Journal for Numerical Methods in Engineering, 52(1-2):121-138, 2001.

[LN02] P. Ladevèze and A. Nouy. A multiscale computational method with time and space homogenization. Comptes-Rendus de l'Académie des Sciences, 330:683-689, 2002.

[LNL02] P. Ladevèze, A. Nouy, and O. Loiseau. A multiscale computational approach for contact problems. Computer Methods in Applied Mechanics and Engineering, 191:4869-4891, 2002.

[LP01] P. Ladevèze and J. P. Pelle. La maîtrise du calcul en Mécanique linéaire et non-linéaire. Hermès Sciences Publications, 2001.

[LSS91] R. W. Lewis, B. A. Schrefler, and L. Simoni. Coupling versus uncoupling in soil consolidation. International Journal for Numerical and Analytical Methods in Geomechanics, 15:533-548, 1991.

[MSV96] R. Matteazzi, B. Schrefler, and R. Vitaliani. Comparisons of partitioned solution procedures for transient coupled problems in sequential and parallel processing, pages 351-357. Advances in Computational Structures Technology. Civil-Comp Ltd, Edinburgh, Scotland, 1996.

[MT02] Y. Maday and G. Turinici. A parareal in time procedure for the control of partial differential equations. Comptes-Rendus de l'Académie des Sciences, 335(Issue 4):387-392, 2002.

[OM95] R. Ohayon and J.-P. Morand. Fluid-Structure Interaction: Applied Numerical Methods. John Wiley \& Sons, 1995.

[PFL95] S. Piperno, C. Farhat, and B. Larrouturou. Partitioned procedures for the transient solution of coupled aeroelastic problems. Part I: model problem, theory and two-dimensional application. Computer Methods in Applied Mechanics and Engineering, 124:79-112, 1995.

[SL98] B. A. Schrefler and R. W. Lewis. The Finite Element Method in the Static and Dynamic Deformation and Consolidation of Porous Media. Wiley, 2nd edition, 1998.

[TS93] E. Turska and B. A. Schrefler. On convergence conditions of partitioned solution procedures for consolidation problems. Computer Methods in Applied Mechanics and Engineering, 106:51-63, 1993.

[TS94] E. Turska and B. A. Schrefler. On consistency, stability and convergence of staggered solution procedures. Rend. Mat. Acc. Lincei, 5:265-271, 1994.

[VA81] P. A. Vermeer and A.Veruijt. An accuracy condition for consolidation by finite elements. International Journal for Numerical and Analytical Methods in Geomechanics, 5:1-14, 1981. 
[YF02] Q. Yu and J. Fish. Temporal homogenization of viscoelastic and viscoplastic solids subjected to locally periodic loading. Computational Mechanics, 29:199-211, 2002.

[ZQTN86] O. C. Zienkiewicz, S. Qu, R. L. Taylor, and S. Nakazawa. The patch test for mixed formulations. International Journal for Numerical Methods in Engineering, 23:1873-1883, 1986. 